\title{
COMPORTAMENTO ALIMENTAR: UM ESTUDO DA RELAÇÃO ENTRE IMC DE CRIANÇAS E SUA PERCEPÇÃO SOBRE AS PRÁTICAS ALIMENTARES PARENTAIS
}

\author{
Nádia Prazeres Pinheiro-Carozzo \\ Universidade Federal do Maranhão \\ Jena Hanay Araujo de Oliveira \\ Universidade Federal do Maranhão
}

\begin{abstract}
Resumo
Práticas alimentares parentais são estratégias utilizadas pelos pais na educação alimentar do filho, buscando o aumento de respostas adequadas e a diminuição de inadequadas. $O$ objetivo deste estudo foi investigar a relação entre a percepção dos filhos sobre tais práticas e o seu índice de massa corporal - IMC. A pesquisa foi transversal e correlacional, contou com amostra de 114 crianças, de 8 a 11 anos, incluídas via amostragem não-probabilística, sendo a participação voluntária e anônima mediante a assinatura do Termo de Consentimento Livre e Esclarecido e do Termo de Assentimento. As crianças foram pesadas, medidas e responderam o Questionário de Verificação das Práticas Alimentares - Versão Filhos (QVPA). Foram encontradas diferenças significativas nas práticas alimentares parentais segundo o IMC da criança: pressão para comer $(p \leq 0,01)$ e restrição alimentar por peso $(p \leq 0,05)$. A pesquisa demonstrou que há relação entre índice de massa corporal do filho e a prática alimentar a ser utilizada pelos pais.

Palavras-chave: práticas alimentares parentais; índice de massa corporal; infância.
\end{abstract}

\section{EATING BEHAVIOR: A RESEARCH CONCERNING THE RELATION BETWEEN CHILDREN'S BMI AND THEIR PERCEPTION ABOUT PARENTAL FEEDING PRACTICES}

\begin{abstract}
Parental feeding practices are strategies used by parents while educating child's eating behavior, aiming to increase appropriate behaviors and reduce inappropriate ones. The aim of this study was to investigate the relationship between children's perception of their parental feeding practices and their body mass index - BMI. The survey was cross-sectional and correlational type. Consisted of 114 children, 8-11 years, included via non-probability sampling, with the voluntary and anonymous participation by signing the Informed Consent Form and the Statement of Consent. Children were weighed, measured and answered the Comprehensive Feeding Practices Questionnaire - Children Version (CFPQ). There were significant differences in parental feeding practices according to the BMI of the child: pressure to eat $(p \leq 0.01)$ and dietary restriction for weight $(p \leq 0.05)$. The research showed that there is a relation between the children's BMI and the feeding practices to be used by parents.

Keywords: parental feeding practices; body mass index; childhood.
\end{abstract}




\title{
COMPORTAMIENTO ALIMENTAR: UN ESTUDIO DE LA RELACIÓN ENTRE EL IMC DE CRIANZAS Y SU PERCEPCIÓN ACERCA DE LAS PRÁCTICAS ALIMENTARIAS PARENTALES
}

\begin{abstract}
Resumen
Prácticas alimentarias parentales son estrategias utilizadas por los padres para la enseñanza de comportamientos alimentarios de los hijos, con el objetivo de aumentar las respuestas adecuadas y disminuir las inadecuadas. El objetivo de este estudio fue investigar la relación entre la percepción del hijo acerca de las prácticas de sus padres y su estado nutricional. El estudio fue trasversal y analítico, compuesto por 114 chicos, de 8 a 11 años, incluidos por amostraje no probabilística, siendo la participación voluntaria y anónima tras la firma del Consentimiento Libre y Esclarecido y el Término de Asentimiento. Los chicos fueron pesados, medidos y contestaron al Cuestionario de Verificación de Prácticas Alimentarias. Se encontraron diferencias significativas en las prácticas alimentarias parentales según el IMC del chico: presión para comer ( $p \leq$ $0,01)$ y restricción alimentarias por el peso $(p \leq 0,05)$. La investigación demuestra que hay relación entre el IMC del hijo y la práctica alimentaria parental a ser utilizada por los padres.
\end{abstract}

Palabras clave: prácticas alimentarias parentales; índice de masa corporal; infancia.

\section{INTRODUÇÃO}

As práticas educativas parentais são comportamentos ou estratégias usadas pelos pais para extinguir comportamentos inadequados, incentivar a ocorrência de comportamentos adequados em seus filhos e para a instalação de princípios morais e habilidades que garantam disciplina, independência, autonomia, responsabilidade, habilidades sociais e bom relacionamento em grupo (Alvarenga, 2001; Alvarenga, Piccinini, Frizzo, Lopes \& Tudge, 2009; Gomide, 2001, 2006).

Nas palavras de Salvador e Weber (2005), as práticas educativas parentais:

[...] correspondem a comportamentos definidos por conteúdos específicos e por objetivos de socialização; diferentes práticas parentais podem ser equivalentes para um mesmo efeito no filho. As práticas são estratégias com o objetivo de suprimir comportamentos considerados inadequados ou de incentivar a ocorrência de comportamentos adequados (Salvador \& Weber, 2005, p. 342).

Especificamente na aprendizagem do comportamento alimentar, as práticas educativas podem ser verificadas em: estratégias para controle do que e quanto os filhos comem, para orientar quanto à alimentação, assim como também podem ser utilizadas estratégias que podem desencadear comportamento alimentar inadequado, como o uso de comida como recompensa e como regulador emocional (Musher-Einzmen \& Holub, 2007). Essas são as denominadas práticas alimentares parentais, que por sua vez, são práticas educativas parentais específicas do contexto alimentar.

As doze práticas alimentares parentais são: 1) controle da alimentação feito pela criança; 2) uso de comida para o controle das emoções; 3) incentivo ao equilíbrio e variedade alimentícia; 4) ambiente com presença de alimentos saudáveis; 5) alimento como recompensa; 6) envolvimento; 7) modelo; 8) 
monitoramento; 9) pressão para comer; 10) restrição alimentar por saúde; 11) restrição alimentar para controle do peso; e 12) ensino sobre nutrição (MusherEizenman \& Holub, 2007). Há práticas consideradas positivas/adequadas, outras negativas/inadequadas e outras que, dependendo do contexto, podem ser positivas ou negativas.

O manejo das práticas alimentares pelos pais é uma das variáveis implicadas na educação alimentar infantil, que por sua vez incide diretamente no controle do peso da criança, uma vez que o ganho ou a perda de peso dependem da ingestão alimentar - aliado a fatores genéticos e de perda calórica influenciando, portanto, no desenvolvimento das condições nutricionais: baixo peso, peso adequado, sobrepeso e obesidade.

Atualmente, a condição nutricional foco da atenção da saúde pública é a obesidade. Ela é definida pela Organização Mundial da Saúde (OMS) como uma patologia nutricional que se caracteriza pela acumulação excessiva de gordura, de maneira que a saúde de tal indivíduo pode ser adversamente afetada com diversos problemas de saúde, incluindo diabetes tipo II, doenças cardiovasculares, hipertensão e algumas formas de câncer (Organização Mundial de Saúde [OMS], 2000).

A incidência de sobrepeso e obesidade em crianças tem aumentado, o que faz com que esse agravo seja considerado, atualmente, uma epidemia em países em desenvolvimento. No Brasil, os números indicam que a população infantil acima do peso vem aumentando. Em 2002, a prevalência de obesidade em crianças alcançava $8,2 \%$ no Nordeste do Brasil e $11,9 \%$ no Sudeste, enquanto que em adolescentes variava entre $6,6 \%$ e $8,4 \%$, respectivamente (Abrantes, Lamounier \& Colosimo, 2002); em 2003, o Instituto Brasileiro de Geografia e Estatística (IBGE) apontou que $16,7 \%$ dos pré-adolescentes e adolescentes brasileiros estavam com excesso de peso (Instituto Brasileiro de Geografia e Estatística [IBGE], 2003); em 2008, dados da Pesquisa de Orçamentos Familiares 2008-2009 (IBGE, 2009), indicaram que, nos últimos 20 anos, os casos de obesidade mais do que quadruplicaram entre crianças de 5 a 9 anos, chegando a $16,6 \%$ (meninos) e $11,8 \%$ (meninas). $E$, mais especificamente, em estudo recente realizado em São Luís - MA, encontrou-se que $21 \%$ das crianças entre oito e 12 anos apresentam sobrepeso e 5,8\%, obesidade (Pinheiro \& Jiménez, 2010).

Com isso, estudar as práticas alimentares parentais e sua relação com o peso da criança pode lançar indicativos de como prevenir os índices alarmantes de sobrepeso e obesidade infantis. O objetivo deste estudo foi investigar as relações existentes entre as práticas alimentares parentais percebidas pelos filhos e o seu índice de massa corporal, que é um método convencional para avaliação do estado nutricional. Segundo Acuña e Cruz (2004), os métodos convencionais são aqueles tradicionalmente usados tanto na prática clínica 
quanto em estudos epidemiológicos, dado sua praticidade, baixo custo e por indicarem com certa precisão o estado nutricional do indivíduo.

\section{MÉTODO}

\section{Participantes}

Os participantes da pesquisa foram incluídos via amostragem nãoprobabilística, cuja participação foi voluntária e anônima mediante a assinatura do Termo de Consentimento Livre e Esclarecido (TCLE) pelos pais ou responsáveis e o Termo de Assentimento (TA) pela criança.

Participaram do estudo 114 crianças, entre oito e 11 anos, com média de $9,8$ anos ( $D P=1)$, sendo 63 do sexo feminino (Ver Tabela 1 ).

Tabela 1.

Distribuição da amostra por gênero e idade

\begin{tabular}{ccccccc}
\hline & \multicolumn{2}{c}{ Meninos } & \multicolumn{2}{c}{ Meninas } & \multicolumn{2}{c}{ Total } \\
& $N$ & $\%$ & $N$ & $\%$ & $N$ & $\%$ \\
\hline 8 anos & 7 & 13,8 & 7 & 11,1 & 14 & 12,3 \\
9 anos & 5 & 9,8 & 16 & 25,4 & 21 & 18,4 \\
10 anos & 25 & 49 & 23 & 36,5 & 48 & 42,1 \\
11 anos & 14 & 27,5 & 17 & 27 & 31 & 27,2 \\
\hline Total & 51 & 44,7 & 63 & 55,3 & 114 & 100 \\
\hline
\end{tabular}

\section{Instrumentos e materiais}

Balança Digital Techline com capacidade para $150 \mathrm{~kg}$.

Antropômetro para aferição de altura.

Questionário de Verificação das Práticas Alimentares (QVPA) - Versão Filhos (Mayer, 2011). Este instrumento é uma tradução adaptada do questionário intitulado Comprehensive Feeding Practice Questionnaire (Musher-Eizenman \& Holub, 2007) para avaliar as práticas alimentares parentais na percepção dos filhos. É composto por 49 itens com três opções de resposta: nunca, às vezes e sempre. Cada questão versa sobre a mãe e, em seguida, sobre o pai. O resultado indica se as práticas alimentares parentais são adequadas ou inadequadas.

Procedimentos para coleta de dados

Em dia e horário combinados com coordenação e corpo docente, a pesquisadora compareceu à escola com uma equipe composta por 3 alunas do Curso de Graduação em Nutrição da Universidade Federal do Maranhão, previamente treinadas na aferição de peso e altura. Contingências inerentes à 
escola pública e à escola privada fizeram com que a coleta de dados ocorresse de maneira distinta, conforme especificado abaixo.

\section{Escola Privada}

Todos os alunos que trouxeram o Termo de Consentimento Livre e Esclarecido (TCLE) assinado foram encaminhados, em grupos de 10 a 15, para uma sala destinada à coleta de dados. Logo que acomodados, thes foi lido o Termo de Assentimento (TA), indicando-Ihes onde deveriam assinar caso aceitassem participar da pesquisa e lhes foram dadas instruções de como responder os instrumentos, frisando que não era permitida a comunicação entre os alunos. Nenhuma criança manifestou interesse em desistir do estudo. Diante do assentimento, foram entregues o TA e o QVPA.

Após terminarem de responder os instrumentos, entre 20 e 30 minutos, as crianças foram encaminhadas para a medição, com o set de questionários em mãos, que deveria ser entregue às alunas da graduação. As crianças subiram na balança, descalços ou com meias, com o uniforme da escola, sendo orientados a retirarem qualquer objeto que houvesse nos bolsos. Ficaram com os pés (calcanhares) juntos, em posição ereta e os braços estendidos ao lado do corpo (posição de Frankfort), sendo anotado o valor obtido. Em seguida, subiram na base do antropômetro, e se puseram em posição de Frankfort (arco orbital inferior alinhado em um plano horizontal com o pavilhão auricular), em posição ortostática, com a cabeça ereta, olhando para frente, com o corpo erguido em extensão máxima, com os pés juntos e com as costas no antropômetro, sendo aproximada a parte superior da régua nas suas cabeças e observado e anotado o valor referente à altura. Essas medidas foram registradas no set de questionários em campo específico.

\section{Escola Pública}

A escola solicitou que todos os alunos respondessem os questionários, mesmo aqueles que não houvessem apresentado o TCLE assinado, com intuito de minimizar possíveis sentimentos de exclusão por parte dos não-participantes do estudo. Com isso, após a aplicação, foram excluídos da pesquisa os questionários cujos TCLE não foram assinados. Diante da dificuldade de leitura dos alunos, optou-se por aplicar os questionários em grupos, lendo item por item junto com os alunos.

A coleta nesta escola foi feita em dois momentos distintos. Em um primeiro momento, as pesquisadoras ficavam do lado de fora da sala de aula e os alunos iam saindo um a um para a aferição de peso e altura. Procedimento realizado tal qual descrito e realizado com os alunos da escola privada. No segundo momento, em dia acordado com a professora da turma, a pesquisadora entrou em sala de aula para a aplicação dos instrumentos. Foi explicado às crianças, o objetivo e a importância da pesquisa, bem como lhes foi lido o TA, 
indicando-Ihes onde deveriam assinar caso aceitassem participar da pesquisa, frisando que não era permitida a comunicação entre os alunos. Nenhuma criança manifestou interesse em desistir do estudo. Um modelo de questão foi escrito no quadro, sinalizando como deveria ser feita a marcação: segundo estavam postas linhas e colunas. Em seguida, foram distribuídos o TA e o QVPA. A pesquisadora lia $o$ item e esperava que os alunos respondessem. Este procedimento foi utilizado para completar todo o questionário. Cada turma levou, em média, quatro horas para responder.

\section{Procedimento para análise dos dados}

Após a coleta, procedeu-se à inserção de dados no programa estatístico SPSS (Statistical Package for the Social Sciences) versão 17.0.

Foram realizadas análises descritivas das variáveis idade e gênero do participante, tipo de escola que frequenta (pública ou privada), turno em que estuda, se pratica alguma atividade física e com que frequência. Estas informações serviram para a caracterização da amostra.

Através da opção criação de variável, foi calculado o IMC de cada criança, obedecendo à fórmula: IMC = Peso / (Altura)2. E, via execução de sintaxe, foi classificado o estado nutricional da criança baseada na curva de crescimento da OMS (MS, 2007), obtendo-se seis grupos de IMC: magreza acentuada, magreza, peso adequado, sobrepeso, obesidade e obesidade grave.

Para identificar a ocorrência de crianças abaixo do peso, peso adequado, sobrepeso e obesidade foram realizada análises de frequência.

Em seguida, para avaliar o uso de práticas alimentares parentais adequadas ou inadequadas, os itens dos questionários foram assignados em 12 subescalas, conforme as práticas alimentares parentais. Em seguida, as análises obedeceram aos cálculos propostos e efetivados por Musher-Eizenman e Holub (2007). Assim, somaram-se os itens que compõem cada subescala para gerar um escore para cada uma das práticas. Para o escore total do questionário, são somadas as subescalas de práticas alimentares consideradas adequadas (incentivo ao equilíbrio e variedade, ambiente, envolvimento, modelo, monitoramento e ensino sobre nutrição) e descontados os escores das inadequadas (controle das emoções, alimento como recompensa, pressão, restrição por saúde e restrição para controle do peso). Diante da ausência de ponto de corte, o escore total foi categorizado em alto, médio e baixo a partir do primeiro e terceiro quartis. Para tanto, a amostra foi dividida em quatro grupos de proporções semelhantes entre si, sendo que os dois grupos intermediários foram categorizados juntos como "médio escore", indicando uso tanto de práticas adequadas quanto inadequadas. Os grupos das extremidades foram categorizados como "baixo escore" ou uso majoritariamente de práticas inadequadas e "alto escore" ou uso majoritariamente de práticas adequadas. 
Por último, para verificar se há diferenças entre práticas alimentares parentais em função do IMC da criança foi realizada análise de variância (ANOVA) com o escore total do teste materno e paterno, bem como a prova não paramétrica Kruskal-Wallis com provas post-hoc $\mathrm{C}$ de Dunnet, quando se tratou de cada prática isoladamente.

\section{RESULTADOS}

\section{Sobre o instrumento QVPA}

Neste estudo, o instrumento apresentou $a=0,839$ para o questionário completo, $a=0,725$ para os itens maternos e $a=0,754$ para os itens paternos.

Sobre o perfil do participante

Estudavam em escola privada, 68,4\% da amostra, 67 crianças praticavam atividade física, com a frequência média de 2,45 vezes por semana ( $D P=1,30)$.

$\mathrm{Na}$ amostra em questão, 69 crianças apresentaram peso adequado, 24 sobrepeso, 14 obesidade. Mais especificamente, $60 \%$ apresentaram peso adequado, $21 \%$ sobrepeso, $12 \%$ obesidade, $3 \%$ obesidade grave, $3 \%$ magreza acentuada e $1 \%$ magreza (Ver Figura 1 ).

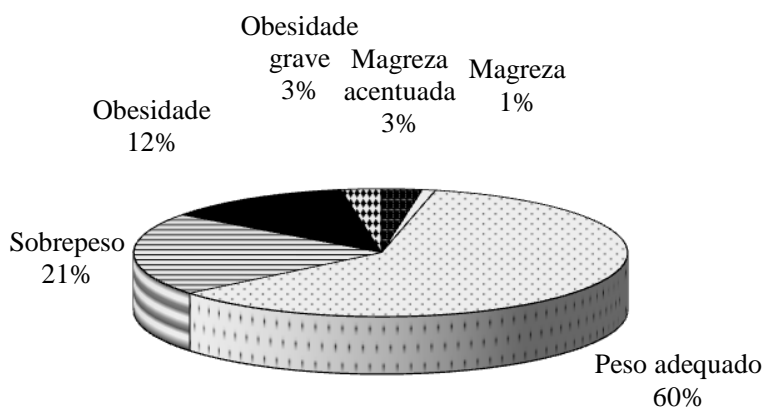

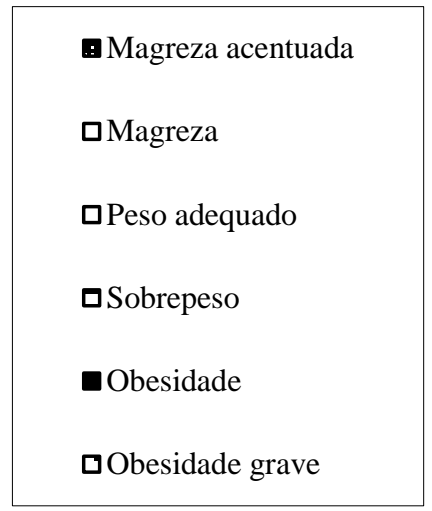

Figura 1. Porcentagem de crianças classificadas segundo o IMC

Sobre o uso de Práticas Alimentares Parentais

Para essa análise, sempre se considera o questionário em duas partes: uma parte composta pelas questões cujo conteúdo versa sobre a mãe e a outra parte sobre o pai.

Ao calcular-se o somatório total do questionário, obtém-se que a média das práticas alimentares maternas é $M=9,23$, $D P=7,26$, já as práticas alimentares paternas apresentou $\mathrm{M}=8,84, \mathrm{DP}=7,68$.

Ao categorizar o escore total em quartis, tem-se o primeiro quartil $\left(\mathrm{Q}_{1 / 4}\right)=$ 4 e o segundo quartil $\left(Q_{2 / 4}\right)=9$, tanto para o pai quanto para a mãe. Por outro lado, o terceiro quartil $\left(\mathrm{Q}_{3 / 4}\right)=15$ para as práticas maternas e $=14,5$ para as 
práticas paternas. Isso equivale dizer para as práticas alimentares maternas que $26,8 \%$ das crianças se encontram no baixo escore, $50 \%$ no médio escore e $23,2 \%$ no alto escore. E para as práticas alimentares paternas, as porcentagens são, respectivamente, 27,8\%; 47,5\%; e 24,7\%. Nesse sentido, não houve diferenças significativas entre as práticas alimentares utilizadas pelo pai e pela mãe, ou seja, os pais apresentam homogeneidade no que tange ao uso de estratégias alimentares. Esses dados podem ser visualizados na figura 2.

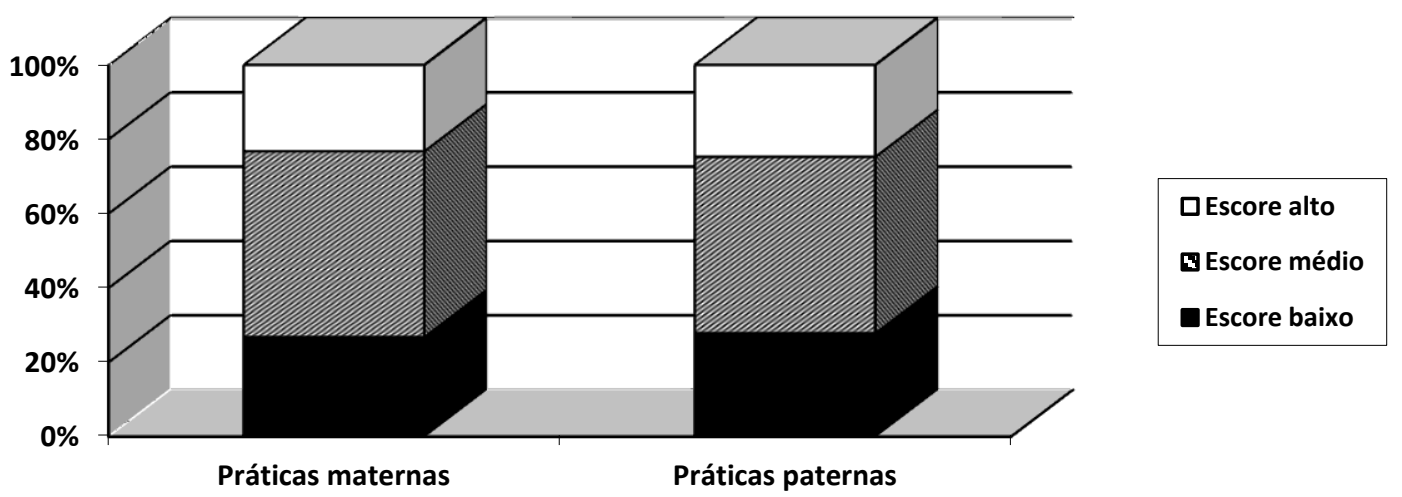

Figura 2. Práticas alimentares parentais segundo escore materno e paterno

Sobre o uso de práticas alimentares parentais segundo o IMC do filho

Através da análise de variância, foi verificado que não há diferença significativa nas práticas alimentares parentais segundo o IMC da criança. Dado que indica que tanto o pai quanto a mãe, não emite estratégias educativas de acordo com o estado nutricional do filho, ou embora a criança apresente magreza, peso adequado, sobrepeso ou obesidade, as práticas escolhidas pelos pais não diferem.

Quando se utiliza cada prática paterna como variável dependente e se analisa a prova de Kruskal-Wallis, pressão para comer é a única prática alimentar com significância. Com um estatístico qui-quadrado de 14,40, com 5 graus de liberdade e significação de 0,013, aceita-se que o uso da prática pressão para comer depende do estado nutricional da criança. A diferença encontra-se entre o grupo de crianças com peso adequado e os grupos com sobrepeso e obesidade grave. O mesmo verifica-se quando a análise é realizada utilizando-se o escore total materno: com um estatístico qui-quadrado de 15,30, com 5 graus de liberdade e significação de 0,010, o uso da prática pressão para comer depende do estado nutricional da criança. A diferença se encontra entre o grupo de criança com peso adequado e os grupos de obesidade e obesidade grave.

Além disso, no caso materno, outra prática alimentar é significativa: restrição alimentar para controle de peso. Este dado, com estatístico quiquadrado de 11,28, 5 graus de liberdade e significação de 0,046, indica que a mãe, ao restringir o consumo de alimentos, leva em consideração o IMC do filho. A diferença encontra-se entre os grupos de peso adequado e obesidade grave. (Ver Tabela 2) 
Tabela 2

Diferenças entre as práticas alimentares segundo o IMC do filho

\begin{tabular}{|c|c|c|c|c|c|c|c|c|c|c|c|c|}
\hline & \multicolumn{2}{|c|}{$\begin{array}{l}\text { Magreza } \\
\text { acentuada }\end{array}$} & \multicolumn{2}{|c|}{ Magreza } & \multicolumn{2}{|c|}{$\begin{array}{c}\text { Peso } \\
\text { adequado }\end{array}$} & \multicolumn{2}{|c|}{ Sobrepeso } & \multicolumn{2}{|c|}{ Obesidade } & \multicolumn{2}{|c|}{$\begin{array}{l}\text { Obesidade } \\
\text { grave }\end{array}$} \\
\hline & $M$ & DP & $M$ & $\mathrm{DP}$ & $M$ & DP & $M$ & DP & $M$ & DP & $M$ & DP \\
\hline $\begin{array}{l}\text { Prática } \\
\text { paterna: } \\
\text { pressão } \\
\text { para } \\
\text { comer }\end{array}$ & 8,33 & 1,52 & 7 & - & $8,14^{\mathrm{ab}}$ & 8,14 & $6,68^{a}$ & 1,88 & 7,25 & 1,65 & $4^{b}$ & - \\
\hline $\begin{array}{c}\text { Prática } \\
\text { materna: } \\
\text { pressão } \\
\text { para } \\
\text { comer }\end{array}$ & 7,66 & 0,57 & 7 & - & $8,25^{\mathrm{cd}}$ & 1,98 & 7,04 & 1,82 & $6,50^{c}$ & 1,60 & $5,33^{d}$ & 2,30 \\
\hline $\begin{array}{c}\text { Prática } \\
\text { materna: } \\
\text { restrição } \\
\text { alimentar } \\
\text { para } \\
\text { controle } \\
\text { de peso }\end{array}$ & 15,66 & 1,15 & 12 & - & $14,82^{d}$ & 2,83 & 16,16 & 3,88 & 16,28 & 3,12 & $19^{d}$ & 1,73 \\
\hline
\end{tabular}

*As médias que compartilham mesmo superíndice apresentam diferença significativa ao nível de 0,05.

\section{DISCUSSÃO}

Neste estudo, foram encontrados índices importantes de sobrepeso e obesidade. No entanto, os números coincidem e reforçam achados da ocorrência de sobrepeso: em Recife (Sintoni, Souza \& Dourado, 2013); no Mato Grosso do Sul (Travi, Bastos \& Pontes, 2011), em Minas Gerais (Lautner, Teles, Costa \& Lautner, 2012), no Rio Grande do Sul (Rech et al., 2010), no Paraná (Mello et al., 2010) e em Santa Catarina (Ribeiro, Fachineto, Pedrozo, Trentin \& Zapoani, 2013). E, se comparado com pesquisa realizada em São Luis no ano de 2010, os dados também coincidem, uma vez que $21 \%$ das crianças entre oito e doze anos apresentavam sobrepeso (Pinheiro \& Jiménez, 2010).

Embora o índice de crianças com sobrepeso tenha se mantido constante, o percentual de crianças obesas ou se manteve constante ou praticamente dobrou: no Mato Grosso do Sul, encontrou-se um índice de $14,4 \%$ de obesidade (Travi et al., 2011) e no Recife de 15,6\% (Sintoni et al., 2013), portanto, similar ao atual. Já em São Luis a ocorrência de obesidade era de 5,8\% (Pinheiro e Jiménez, 2010), em Santa Catarina 5,3\% (Ribeiro et al., 2013), no Rio Grande do Sul, 8\% (Rech et al., 2010) e no Paraná, 7\% (Mello et al., 2010).

No que se refere ao questionário utilizado, QVPA, obteve índices de confiabilidade moderados para um instrumento. Essa confiabilidade dá suporte a que os achados e medições deste estudo estão isentas de erros aleatórios e, por 
conseguinte, se aproximam da variância de valores verdadeiros. Portanto, este instrumento revelou-se um importante instrumento para a investigação das práticas alimentares parentais e, por conseguinte, maior compreensão de como os pais estão educando seus filhos no que se refere ao comportamento alimentar.

Através do QVPA, pode-se perceber que pais e mães não diferem quanto ao uso de práticas alimentares e que tampouco há diferença entre as estratégias educativas utilizadas pelos pais na hora de ensinar comportamentos alimentares. Esse dado é importante, pois demonstra que os pais agem de maneira similar com o filho, sem emitir comandos contrários, evitando assim que o filho discrimine e aja de maneira diferente dependendo do responsável que esteja presente. Coincidindo com literatura que aponta nesse mesmo sentido (Haycraft \& Blisset, 2008). É o que Silva (2000) considera como habilidade social parental adequada: entendimento do casal quanto à educação do filho. Assim, pode-se sugerir que se tem uma amostra de pais socialmente habilidosos no que tange a esse aspecto específico.

Apesar de comandos e regras comuns, percebe-se o uso de práticas alimentares parentais inadequadas em percentual de 26 a $27 \%$. Isto implica que $1 / 4$ dos pais estão utilizando estratégias inadequadas no ensino de comportamentos alimentares de seus filhos. Este é um dado importante, uma vez que as práticas alimentares paternas inadequadas estão relacionadas a práticas alimentares infantis inadequadas, bem como a ocorrência de IMC inadequado (Kiefner-Burmeister, Hoffmann, Meers, Koball \& Musher-Eizenman, 2014; Powell, Farrow \& Meyer, 2011; Rodgers et al., 2013; Taylor, Wilson \& Mohr, 2011).

Por último, a prática alimentar parental pressão para comer alcançou significância em relação ao IMC da criança. A diferença encontra-se entre o grupo de crianças com peso adequado e os grupos com sobrepeso e obesidade grave para os pais; e entre o grupo peso adequado com os grupos de obesidade e obesidade grave para as mães. Assim, na medida em que aumenta o IMC, diminui a pressão sentida para comer. Pode-se supor, portanto, que as crianças por estarem abaixo do peso são pressionadas pelos pais para comer e atingirem o peso normal e as crianças que realmente estão acima do peso, não são pressionadas. Nas práticas paternas, a média das crianças obesas é maior que a média das crianças com sobrepeso, indicando que as crianças obesas sentem mais pressão para comer que as crianças com sobrepeso. Pode-se, então, levantar a hipótese de que os pais as pressionam para comer alimentos mais saudáveis e não qualquer tipo de alimento.

Tais resultados assemelham-se ao de outros estudos onde mães de crianças mais magras pressionam mais seus filhos a comer (Lee \& Birch, 2002; Matheson, Robinson, Varady \& Killen, 2006; Spruijt-Metz, Lindquist, Birch, Fisher \& Goran, 2002), que esta prática é utilizada menos em crianças com sobrepeso 
(Kroller \& Warschburger, 2008) e que pais de crianças com IMC alto utilizam menos prática pressão para comer (Blissett \& Haycraft, 2008).

Sobre as práticas maternas, restrição alimentar por peso apresentou significância em relação ao IMC da criança, indicando que a mãe, ao restringir o consumo de alimentos, leva em consideração o IMC do filho. A maior média de restrição é de crianças com obesidade grave, sendo seguida pelas obesas (em segundo lugar) e pelas com sobrepeso. Mas estatisticamente, a diferença encontra-se entre as crianças com peso adequado e obesidade grave. Este dado foi também encontrada por Mayer (2011), porém, em ambos os pais.

Esse resultado já foi tratado pela literatura e essa prática é apontada como a primeira opção dos pais diante do excesso de peso do filho, como uma tentativa, em curto prazo, de limitar o consumo de alimentos não saudáveis (Birch, Fischer \& Davidson, 2003). Porém, em longo prazo, é classificada como uma prática perigosa, pois a criança aprende o comportamento de escolha de alimentos e quantidade de comida ingerida a partir das regras parentais e não, conforme seus conhecimentos sobre nutrição ou estímulos indicadores de fome e saciação. Ao mesmo tempo em que, quando a criança estará privada de determinados alimentos, a probabilidade de ela empenhar-se em comportamentos, situações ou pessoas que possam suprir sua privação fica aumentada. Nas palavras de Skinner, "se conhecermos o nível de privação (...) poderemos prever com maior precisão quão reforçador será determinado acontecimento e qual a probabilidade de uma pessoa empenhar-se em comportamento pertinente" (Skinner, 2002, p. 46). Uma consequência pode ser que alimentos não saudáveis passam a ser poderosos reforçadores e foco de atenção da criança sempre que ela está longe do restritor, seja ele pai ou mãe.

Este dado foi encontrado em estudos realizados, inclusive fora do país. Um estudo comparativo entre Estados Unidos e França (Musher-Eizenman, De Lauzon-Guillain, Holub, Leporc \& Charles, 2009), destacou que nos dois países, alto IMC das crianças está relacionado com maior restrição alimentar por saúde e restrição alimentar por peso. E também, um estudo longitudinal australiano revela que as mães utilizam mais práticas como controle emocional por comidas, restrições de ambos os tipos, incentivo a variedade na medida em que verifica que seu filho vai ganhando mais peso que aquele indicado para sua idade (Rodgers et al., 2013).

Diante do encontrado nesta investigação, faz-se imprescindível continuar estudando a relação entre práticas alimentares parentais e o estado nutricional de crianças, utilizando-se para tanto, o instrumento ora usado, bem como outros instrumentos. As limitações dessa pesquisa incluem o uso de um tamanho amostral reduzido. Ademais desta, questiona-se se o fato de que o procedimento de coleta de dados tenha sido feito de formas diferentes nas escolas haja gerado vieses nas respostas ao instrumento, uma vez que os alunos da escola pública viram seu peso marcado na balança antes de completarem o instrumento. 
Como encaminhamento para futuros estudos, sugere-se calcular estado nutricional de pai e mãe, verificando se este desempenha papel relevante na compreensão do problema; utilizar, além do IMC, outra medida antropométrica indicadora de estado nutricional, tais como circunferências, medidas de dobras e pregas cutâneas, comprimento de segmento, largura óssea, compleição, dentre outros; e aumentar o tamanho da amostra.

\section{REFERÊNCIAS}

Abrantes, M. M., Lamounier, J. A., \& Colosimo, E. A. (2002). Prevalência de sobrepeso e obesidade em crianças e adolescentes nas regiões Nordeste e Sudeste. Jornal de Pediatria, 78(4), 335-340. doi: 10.1590/S002175572002000400014

Acuña, K. \& Cruz, T. (2004). Avaliação do estado nutricional de adultos e idosos e situação nutricional da população brasileira. Arquivos Brasileiros de Endocrinologia \& Metabologia, 48(3), 35-361. doi: 10.1590/S000427302004000300004

Alvarenga, P. (2001). Práticas educativas parentais como forma de prevenção de problemas de comportamento. In: H. J. Guilhardi (Org.), Sobre Comportamento e Cognição (vol. 8, pp. 54-60). Santo André: ESETec Editores Associados.

Alvarenga, P., Piccini, C. A., Frizzo, G. B., Lopes, R. S., \& Tudge, J. (2009). Estabilidade e mudanças nas práticas educativas de mães e pais dos 18 para os 24 meses de vida da criança. Interação, 13(2), 253-262. Recuperado de: http://ojs.c3sl.ufpr.br/ojs/index.php/psicologia/article/viewFile/12719/1137 1

Birch, L. L., Fisher, J. O., \& Davison, K. K. (2003). Learning to overeat: maternal use of restrictive feeding practices promotes girls' eating in the absence of hunger. American Journal of Clinical Nutrition, 78(2), 215-220. Recuperado de: http://ajcn.nutrition.org/content/78/2/215.full.pdf+html

Blissett, J., \& Haycraft, E. (2008). Are parenting style and controlling feeding practices related? Appetite, 50(2-3), 477-485. doi: 10.1016/j.appet.2007.10.003

Gomide, P. I. C. (2001). Efeito das práticas educativas no desenvolvimento do comportamento anti-social. In M. L. Marinho \& V. E. Caballo (Orgs.), Psicologia clínica e da saúde. Londrina: UEL.

Gomide, P.I. C. (2006). Inventário de Estilos Parentais. Modelo teórico: manual de aplicação, apuração e interpretação. Petrópolis, RJ: Vozes.

Haycraft, E. L., \& Blissett, J. M. (2008). Maternal and paternal controlling feeding practices: reliability and relationships with BMI. Obesity, 16(7), 1552-1558. doi: $10.1038 /$ oby.2008.238 
Instituto Brasileiro de Geografia e Estatística. (2003). Pesquisa Nacional por Amostras de domicílio. Recuperado em 10 março de 2013, de http://www.ibge.gov.br.

Instituto Brasileiro de Geografia e Estatística. (2009). Pesquisa de Orçamentos Familiares 2008-2009. Recuperado em 10 março de 2013, de http://www.ibge.gov.br.

Kiefner-Burmeister, A. E., Hoffmann, D. A., Meers, M. R., Koball, A. M., \& Musher-Eizenman, D. R. (2014). Food consumption by young children: a function of parental feeding goals and practices. Appetite, 74(1), 6-11. doi: 10.1016/j.appet.2013.11.011

Kroller, K., \& Warschburger, P. (2008). Associations between maternal feeding style and food intake of children with a higher risk for overweight. Appetite, 51(1), 166-172. doi: 10.1016/j.appet.2008.01.012

Lautner, R. Q., Teles, J. A., Costa, K. M., \& Lautner, M. A. F. A. (2012). Índice de prevalência de obesidade e hipertensão em crianças e pré-adolescentes no ensino público de Formiga, MG. Enfermagem Revista, 15(3), 297-307. Recuperado de: http://periodicos.pucminas.br/index.php/enfermagemrevista/article/view/5 $172 / 5180$

Lee, Y., \& Birch, L. L. (2002). Diet quality, nutrient intake, weight status, and feeding environments of girls meeting or exceeding the american of pediatrics recommendations for total dietary fat. Minerva Pediatrics, 54(3), 179-186. doi: 10.1542/peds.107.6.e95

Matheson, D. M., Robinson, T. N., Varady, A., \& Killen, J. D. (2006). Do MexicanAmerican mothers' food-related parenting practices influence their children's weight and dietary intake? Journal of the American Dietetic Association, 106(11), 1861-1865. Recuperado de: http://www.sciencedirect.com/science/article/pii/S0002822306018281

Mayer, A. P. F. (2011). As relações entre família, escola e o desenvolvimento do sobrepeso e obesidade infantil. Dissertação de Mestrado, Universidade Federal do Paraná, Curitiba, Paraná, Brasil.

Mello, A. D. M., Marcon, S. S., Hulsmeyer, A. P. C. R., Cattai, G. B. P., Ayres, C. S. L. S., \& Santana, R. G. (2010). Prevalência de sobrepeso e obesidade em crianças de 6 a 10 anos de escolas municipais de área urbana. Revista Paulista de Pediatria, 28(1), 48-54. doi: 10.1590/S010305822010000100009

Musher-Eizenman, D. R., \& Holub, S. C. (2007). Comprehensive feeding practices questionnaire: validation of a new measure of parental feeding practices. Journal of Pediatric Psychology, 32(8), 960-972. doi: 10.1093/jpepsy/jsm037 
Musher-Eizenman, D. R., De Lauzon-Guillain, B., Holub, S. C., Leporc, E., \& Charles, M. A. (2009). Child and parent characteristics related to parental feeding practices. A cross-cultural examination in the US and France. Appetite, 52(1), 89-95. doi: 10.1016/j.appet.2008.08.007

Organização Mundial de Saúde. (2000). La OMS difunde un nuevo patrón de crecimiento infantil. Recuperado em 20 março de 2013, de http://www.who.int/mediacentre/news/releases/2006/pr21/es/index.html.

Pinheiro, N., \& Jiménez, M. (2010). Percepção e Insatisfação corporal: um estudo com crianças brasileiras. Revista Psico, 41(4), 510-516. Recuperado de: http://revistaseletronicas.pucrs.br/ojs/index.php/revistapsico/article/view/7 $358 / 5960$

Powell, F. C., Farrow, C. V. \& Meyer, C. (2011). Food avoidance in children: the influence of maternal feeding practices and Behaviours. Appetite, 57(3), 683-692. doi: 10.1016/j.appet.2011.08.011

Rech, R. R., Halpern, R., Constanzi, C. B., Bergmann, M. L. A., Alli, L. R., Matos, A. P., et al (2010). Prevalência de sobrepeso e obesidade em escolares de 7 a 12 anos de uma cidade Serrana do RS, Brasil. Revista Brasileira de Cineantropometria \& Desempenho Humano, 12(2), 90-97. Recuperado de: http://www.scielo.br/pdf/rbcdh/v12n2/a02v12n2

Ribeiro, A. J. P., Fachineto, A., Pedrozo, S. C., Trentin, A. P., \& Zapoani, C. (2013). Prevalência de sobrepeso e obesidade em escolares com idade de 7 a 17 anos residentes nos municípios pertencentes à Secretaria de Desenvolvimento Regional de São Miguel do Oeste/SC. Revista da Faculdade de Educação Física da UNICAMP (Campinas), 11(1), 57-73. Recuperado de: http://fefnet178.fef.unicamp.br/ojs/index.php/fef/article/view/717/pdf

Rodgers, R. F., Paxton, S. J., Massey, R., Campbell, K. J., Wertheim, E. H., Skouteris, H., et al (2013). Maternal feeding practices predict weight gain and obesogenic eating behaviors in young children: a prospective study. International Journal of Behavioral Nutrition and Physical Activity, 10(24), 1-10. doi: 10.1186/1479-5868-10-24

Salvador, A. P. V., \& Weber, L. N. D. (2005). Práticas Educativas Parentais: um estudo comparativo da interação familiar de dois adolescentes distintos. Interação em Psicologia, 9(2), 341-353. Recuperado de: http://ojs.c3sl.ufpr.br/ojs/index.php/psicologia/article/viewFile/4782/3669

Silva, A. T. B. (2000). Problemas de comportamento e comportamentos socialmente adequados: sua relação com as atividades educativas de pais. Dissertação de Mestrado, Universidade Federal de São Carlos, São Carlos, São Paulo, Brasil. 
Sintoni, F., Souza, D. M. T. de, \& Dourado, A. C. M. (2013). Prevalência de sobrepeso e obesidade em estudantes de uma escola do Recife - PE. Anais, XII Congresso Brasileiro de Medicina de Família e Comunidade (p. 854). Belém, PA: Sociedade Brasileira de Medicina de Família e Comunidade.

Skinner, B. F. (2002). Sobre o Behaviorismo. São Paulo: Cultrix.

Spruijt-Metz, D., Lindquist, C. H., Birch, L. L., Fisher, J. O., \& Goran, M. I. (2002). Relation between mother's child-feeding practices and children's adiposity. American Journal of Clinical Nutrition, 75(3), 581-586. Recuperado de: http://ajcn.nutrition.org/content/75/3/581.full.pdf+html

Taylor, A., Wilson, C., Slater, A., \& Mohr, P. (2011). Parent- and child-reported parenting. Associations with child weight-related outcomes. Appetite, 57(3), 700-706. doi: 10.1016/j.appet.2011.08.014

Travi, M. I. C., Bastos, P. R. H. de O., \& Pontes, E. R. J. C. (2011). Prevalência de sobrepeso, obesidade e circunferência abdominal alterada em escolares de 6 a 11 anos de idade em Campo Grande/MS. Revista Brasileira em Promoção da Saúde, 24(1), 54-62. Recuperado de: http://www.scielo.br/scielo.php?pid=S1413$81232013000500027 \&$ script $=$ sci_arttext

Sobre os autores

Nádia Prazeres Pinheiro-Carozzo é mestra em Psicologia pela Universidade Federal do Maranhão. Docente do Departamento de Psicologia da Universidade Federal do Maranhão - UFMA. nadia.pinheiro@ufma.br Jena Hanay Araujo de Oliveira é mestra em Psicologia Clínica e doutora em Psicologia como Profissão e Ciência pela PUC-Campinas. Docente do Departamento de Psicologia e do Programa de Pós-Graduação em Psicologia da Universidade Federal do Maranhão - UFMA. jenahanay@yahoo.com

Recebido em: 20/10/2014

Revisado em: 02/03/2015 Aceito em: 08/04/2015 